\title{
Avaliação do Kt para estimativa da evapotranspiração de referência (ETo) em Campos dos Goytacazes, RJ
}

\author{
Barbara dos S. Esteves', José C. Mendonçą2, Elias F. Sousa ${ }^{3}$ \& Salassier Bemardo 3
}

\begin{abstract}
RESUMO
Neste trabalho foram avaliados diferentes métodos de determinação do coeficiente do tanque (Kt), para a estimativa diária da evapotranspiração de referência (ETo) utilizando o método do tanque Classe A (TCA). Foram avaliadas as metodologias propostas por Allen et al. (1998), Bernardo et al., (1996), Cuenca (1989) e Snyder (1992); os valores da ETo diária estimados foram correlacionados com os estimados pelo método de Penman-M onteith parametrizado (FAO -56). Para a estimativa da ETo foi utilizado uma série histórica de 10 anos de dados coletados em uma estação automática, modelo Thies Clima, instalada na Estação Evapotranspirométrica da Universidade Estadual do Norte Fluminense Darcy Ribeiro (UENF). Os resultados obtidos, considerando-se as diferentes metodologias para o cálculo do Kt, mostrou um bom desempenho do método do tanque Classe A para a estimativa de valores diários da ETo (coeficientes $\mathrm{R}^{2}>0,79 \mathrm{e}$ $D>0,90)$. As metodologias propostas por Cuenca (1989), Bernardo et al. (1996) e Allen (1998) apresentaram o mesmo índice de concordância $(D=0,95)$. 0 menor erro médio absoluto $(E M A=0,50)$ foi obtido pelo método de Cuenca (1989) que também mostrou a maior eficiência $(E F=0,81)$.
\end{abstract}

Palavraschave: manejo de irrigação, Penman-Monteith, agrometeorologia

\section{Evaluation of "Class A" pan coefficients to estimate the referential evapotranspiration in Campos dos Goytacazes, RJ}

\begin{abstract}
In this work, different methods of determination of pan coefficient $(\mathrm{Kp})$ for a daily estimation of referential evapotranspiration (ETo) using the "Class A" pan method (TCA) were analyzed. Methodologies proposed by Allen et al. (1998), Bernardo et al. (1996), Cuenca (1989) and Snyder (1992) were evaluated, and values of daily ETo estimated using TCA were compared to ones estimated by Penman-M onteith (FAO-56). A ten years historical series of data collected from an automatic station, Thies Clima model, was used installed at evapotranspiration station of U niversidade Estadual do Norte Fluminense (UENF). A good fulfillment of Class A pan to estimate daily values of ETo by different ways to calculate Kp ( $R^{2}>0.79$ and $D>0.90$ ) was verified, with Cuenca (1989), Bernardo et al. (1996), and Allen (1998) methods showing the same concordance index $(D=0.95)$. The smallest absolute mean error $(0.50)$ was showed by Cuenca (1989) method, which also presented the highest efficiency $(E F=0,81)$.
\end{abstract}

Key words irrigation management, Penman-M onteith, agrometeorology

\footnotetext{
1 Engenheira Agrônoma, Mestranda em Engenharia Agrícola, LEAG/U EN F, Av. Alberto Lamego 2000 - Pq. Califórnia. CEP 28015-620, Campos dos Goytacazes, RJ. Fone: (22) 2739-7286. E-mail: barbbarase@yahoo.com.br

2 LAMET/UENF. Fone: (22) 2736-6501. E-mail: mendonca@uenf.br

3 LEAG/UENF. Fone: (22) 2739-7286. E-mail: efs@uenf.br; salassie@uenf.br
} 


\section{INTRODUÇÃO}

Na agricultura a irrigação é uma técnica fundamental para se evitar o risco de déficit hídrico nas culturas, aumentar a produtividade e intensificar o uso da terra; por outro lado, o elevado volume de água requerido nesta prática exige um uso de forma racional. A determinação da quantidade de água necessária para atender à demanda das culturas irrigadas, aliada a um correto dimensionamento do sistema de irrigação é o principal parâmetro para o uso sustentável dos recursos hídricos na agricultura irrigada.

No que se refere ao manejo de irrigação, a base para a quantificação da água a ser aplicada a uma determinada cultura está comumente associada à capacidade da superfície do solo e da vegetação de perder água para a atmosfera. A forma usual de se quantificar a água a ser aplicada ao longo do ciclo da cultura, é considerar os processos de evaporação do solo e de transpiração da planta conjuntamente, no que se denomina evapotranspiração (Silva \& Rao, 2006). Borges \& Mediondo (2007) definem evapotranspiração de referência (ETo) como o processo de perda de água para a atmosfera por meio de uma superfície padrão gramada, cobrindo a superfície do solo e sem restrição de umidade.

São diversos os métodos para a estimativa da ETo, isto é, métodos diretos ou indiretos. Encontram-se na literatura especializada, diversos métodos que propiciam a estimativa do consumo hídrico de culturas, porém sua utilização é bastante limitada com propósitos práticos, face à ausência de técnicas apropriadas que viabilizem a estimativa da evapotranspiração, de forma simples e confiável, de acordo com a disponibilidade dos parâmetros relacionados à planta, ao solo e à atmosfera (Silva et al., 2005). Segundo Braga et al. (2008), o tanque Classe A é um dos métodos indiretos de uso generalizado, inclusive no Brasil, em virtude do seu fácil manejo e baixo custo de implantação. Dentre os métodos diretos os aparelhos mais utilizados são os lisimetros, cujo uso é restringido a instituições de pesquisa devido, principalmente, ao seu grande custo de implantação e operacional.

O tanque Classe A (TCA) foi desenvolvido pelo Serviço Meteorológico Norte-Americano (U.S.W.B.) oferecendo uma estimativa dos efeitos combinados da radiação solar, do vento, da temperatura e da umidade relativa do ar. Quando bem conduzido, este método oferece resultados confiáveis na determinação da evapotranspiração de referência (Oliveira et al., 2008).

Diversos pesquisadores questionam o método de escolha do Kt para estimação da ETo pelo método do tanque Classe A. O coeficiente adotado para determinada região deve ser adequado para que não haja estimativas equivocadas; por esta razão, a pesquisa regional em busca de valores de Kt específicos é de suma importância para o manejo racional da água.

Dessa forma este estudo teve por objetivo avaliar diferentes metodologias para a determinação do coeficiente do tanque (Kt), utilizado no método do tanque Classe A para a estimativa da evapotranspiração de referência (ETo) em escala diária, no município de Campos dos Goytacazes, RJ.

\section{MATERIAL E MÉTODOS}

Para o desenvolvimento deste trabalho utilizou-se uma série de 10 anos de dados meteorológicos diários (período de 1996 a 2006) coletados por uma estação automática, modelo Thies Clima, instalada na Estação Experimental de Campos dos Goytacazes, Pesagro-Rio (coordenadas geográficas de $21^{\circ} 18^{\prime} 47^{\prime}$ ' de latitude Sul e $41^{\circ} 18$ ' 24 " de longitude Oeste e altitude de $11 \mathrm{~m}$ ), pertencente à Universidade Estadual do Norte Fluminense Darcy Ribeiro (UENF).

Excluíram-se os dados referentes aos dias de chuva e um dia a estes subsequente, irrigação do gramado, manutenção de equipamentos e demais anormalidades.

A ETo diária considerada padrão de referência foi estimada pelo método de Penman-Monteith parametrizado, proposto por Allen et al. (1998), de acordo com a Eq. 1:

$$
\begin{aligned}
\mathrm{ETo}^{\mathrm{PM}}= & 0,408 \Delta(\mathrm{Rn}-\mathrm{G})+\gamma(900 / \mathrm{T}+273) \mathrm{U}_{2}(\mathrm{es}-\mathrm{ea}) / \Delta \\
& +\gamma\left(1+0,34 \mathrm{U}_{2}\right)
\end{aligned}
$$

em que:

ETo - evapotranspiração potencial, em $\mathrm{mm} \mathrm{d}^{-1}$

$\mathrm{Rn}$ - saldo de radiação, em $\mathrm{MJ} \mathrm{m}^{-2} \mathrm{~d}^{-1}$

$\mathrm{G}$ - fluxo de calor no solo, em $\mathrm{MJ} \mathrm{m}^{-2} \mathrm{~d}^{-1}$

$\mathrm{T}$ - temperatura média diária do $\mathrm{ar}$, em ${ }^{\circ} \mathrm{C}$

$\gamma$ - constante psicrométrica, em $\mathrm{kPa}^{\circ} \mathrm{C}^{-1}$

$\Delta$ - tangente da curva de pressão de saturação de vapor em função da temperatura do ar em $\mathrm{kPa}{ }^{\circ} \mathrm{C}^{-1}$

$\mathrm{U}_{2}$ - velocidade média diária do vento a $2 \mathrm{~m}$ de altura, em $\mathrm{m} \mathrm{s}^{-1}$

(es-ea) - déficit de pressão de vapor, em kPa

Para a estimativa da ETo pelo método do TCA utilizouse a Eq. 2:

$$
\mathrm{ETo}_{\mathrm{TCA}}=\mathrm{EV} \mathrm{Kt}
$$

em que:

$\mathrm{EV}$ - evaporação do tanque classe $\mathrm{A}$, em $\mathrm{mm} \mathrm{d}^{-1}$

$\mathrm{Kt}$ - coeficiente do tanque (adimensional)

Os valores de Kt diários foram determinados pelas seguintes metodologias:

Cuenca (1989) apresentada na Eq. 3

$$
\begin{aligned}
\mathrm{Kt}= & 0,475-2,410^{-4} \mathrm{U}_{2}+5,1610^{-3} \mathrm{H}+1,810^{-3} \mathrm{~F}-1,610^{-5} \mathrm{H}^{2} \\
& -1,0110^{-6} \mathrm{~F}^{2}-810^{-9} \mathrm{H}^{2} \mathrm{U}_{2}-110^{-8} \mathrm{H}^{2} \mathrm{~F}
\end{aligned}
$$

em que:

$\mathrm{U}_{2}$ - velocidade do vento a $2 \mathrm{~m}$ de altura, em $\mathrm{km} \mathrm{d}^{-1}$

$\mathrm{H}$ - umidade relativa média, em percentagem

F - bordadura da área grama, considerada igual a 15 m

Snyder (1992) apresentada na Eq. 4

$\mathrm{Kt}=0,482+0,024 \ln (\mathrm{F})-0,000376 \mathrm{U}_{2}+0,0045 \mathrm{H}$

Bernardo et al. (1996) apresentada na Eq. 5

$$
\mathrm{Kt}=0,69
$$


Allen et al. (1998) apresentada na Eq. 6

$$
\begin{aligned}
\mathrm{Kt}= & 0,108-0,0286 \mathrm{U}_{2}+0,0422 \ln (\mathrm{F})+1434 \ln (\mathrm{H}) \\
& -0,000631[\ln (\mathrm{F})]^{2} \ln (\mathrm{H})
\end{aligned}
$$

Para avaliação da performance dos métodos, procedeu-se a uma análise de regressão linear, considerando-se o modelo linear $\mathrm{y}=\mathrm{a}+\mathrm{bx}$, na qual a variável dependente foi $\mathrm{o}$ método de Penman-Monteith $\left(\mathrm{ETo}^{\mathrm{PM}}\right)$, e a variável independente o método do TCA estimado com as diferentes metodologias de estimativa de Kt. Utilizaram-se, ainda, o índice de concordância de Willmott (D), o erro médio absoluto (EMA), o erro máximo (EMAX) e a eficiência do método (EF), através das Eq. 7, 8, 9 e 10, respectivamente:

$$
\begin{aligned}
& \mathrm{D}=1-\Sigma\left[(\mathrm{O}-\mathrm{E})^{2} / \Sigma(|\mathrm{E}-\mathrm{X}|+|\mathrm{O}-\mathrm{X}|)^{2}\right] \\
& \mathrm{EMA}=1 / \mathrm{n} \Sigma|\mathrm{O}-\mathrm{E}| \\
& \mathrm{EMAX}=\operatorname{MAX}(|\mathrm{O}-\mathrm{E}|)^{\mathrm{n}} \\
& \mathrm{EF}=\left[\Sigma(\mathrm{O}-\mathrm{X})^{2}+\Sigma(\mathrm{O}-\mathrm{E})^{2}\right] / \Sigma(\mathrm{O}-\mathrm{X})^{2}
\end{aligned}
$$

em que:

$$
\begin{aligned}
& \mathrm{O} \text { - valores estimados por } \mathrm{ETo}^{\mathrm{PM}} \\
& \mathrm{E} \text { - valores estimados pelos demais métodos } \\
& \mathrm{X} \text { - média dos valores } \mathrm{ETo}^{\mathrm{PM}}
\end{aligned}
$$

\section{RESULTADOS E DISCUSSÃO}

Na Tabela 1 estão apresentadas as médias mensais da temperatura do ar, da umidade relativa do ar e da velocidade do vento medidas a $2 \mathrm{~m}$ de altura para os dez anos de dados analisados.

O coeficiente do tanque Classe A varia de acordo com o local e as condições climáticas (Allen et al., 1998). Os fatores meteorológicos apresentados na Tabela 1 interferem diretamente na estimativa da ETo pelo método do TCA. A umidade do ar é uma variável dependente da temperatura, atuando indiretamente nos processos evaporativos. À medi-

Tabela 1. Valores médios mensais da temperatura do ar (Tar), umidade relativa média (UR), velocidade do vento a $2 \mathrm{~m}$ (VV) para o período de estudo (1996-2006)

\begin{tabular}{lccc}
\hline Meses & Tar $\left({ }^{\circ} \mathbf{C}\right)$ & UR $(\%)$ & VV $\left.\mathbf{~ ( m ~ s}^{-1}\right)$ \\
Janeiro & 26,47 & 74,35 & 2,36 \\
Fevereiro & 26,54 & 73,55 & 2,12 \\
Março & 25,69 & 75,75 & 1,73 \\
Abril & 24,41 & 76,70 & 1,54 \\
Maio & 21,85 & 75,16 & 1,50 \\
Junho & 20,98 & 77,41 & 1,51 \\
Julho & 20,28 & 76,35 & 1,68 \\
Agosto & 21,60 & 75,90 & 2,24 \\
Setembro & 22,16 & 75,80 & 2,43 \\
Outubro & 23,07 & 75,90 & 2,31 \\
Novembro & 24,29 & 76,19 & 2,34 \\
Dezembro & 25,79 & 75,99 & 2,21 \\
\hline
\end{tabular}

da que a temperatura decresce numa mesma umidade absoluta de ar, haverá aumento na umidade relativa e queda na evaporação. A velocidade do vento é outro fator importante nos processos evaporativos. O aumento da velocidade do vento e da turbulência do ar faz com que haja a remoção da água, transferindo grandes quantidades de ar acima da superfície evaporante, criando uma condição favorável ao aparecimento de um gradiente de pressão de vapor, aumentando o poder evaporativo local.

Na Figura 1 são apresentados os valores médios mensais da evapotranspiração de referência obtida pela metodologia de Penman-Monteith e pelo tanque Classe A utilizando as diferentes metodologias de obtenção do Kt.

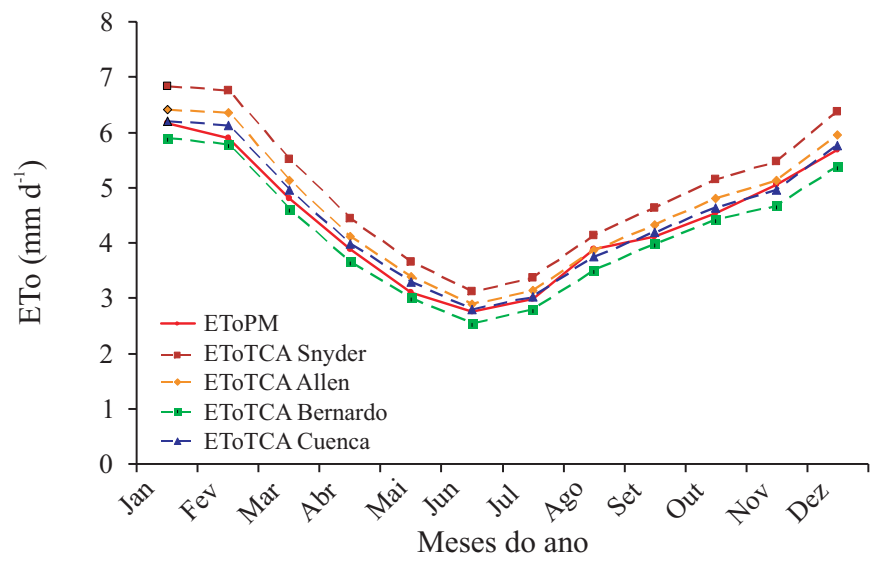

Figura 1. Valores médios mensais da evapotranspiração de referência (ETo) estimada pelo método de Penman-Monteith parametrizado e pelo tanque Classe A com o uso das diferentes metodologias de obtenção do coeficiente do tanque $(\mathrm{Kt})$

Observando a Figura 1 pode-se constatar que as quatro metodologias testadas apresentaram o mesmo ajustamento que a metodologia padrão, para todos os meses do ano. Podese observar na Figura 1, que as metodologias de Cuenca (1989), Allen et al. (1996) e Snyder (1992) superestimaram o método usado como padrão $\left(\mathrm{ETo}^{\mathrm{PM}}\right)$, resultado também obtido por Braga et al. (2008). Duarte et al. (2003) citam que devido à área relativamente pequena do TCA, da absorção da radiação pelas paredes do recipiente e pelo fato dele criar seu próprio ambiente aerodinâmico ao ficar exposto acima do solo, obtêm-se valores de evaporação às vezes um pouco exagerados, proporcionando uma superestimativa da ETo. Os erros de estimativa por parte das metodologias também podem ser atribuídos a maior susceptibilidade ao processo de advecção, a qual o tanque está sujeito. Ainda na Figura 1, pode-se observar que o valor fixo proposto por Bernardo et al. (1996) subestimou a ETo ${ }^{\mathrm{PM}}$. Conceição (2002) também empregou em seu trabalho um valor fixo de Kt utilizado regionalmente e encontrou valores satisfatórios, tal qual neste estudo, observando que fatores como a umidade relativa do ar e a velocidade do vento não devem apresentar grandes variações durante o período analisado.

Na Figura 2 são apresentados os gráficos das correlações entre as estimativas diárias obtidas pelo método do tanque Classe A utilizando as quatro metodologias de obtenção do 
Kt e a estimada pelo método de Penman Monteith (ETo $\left.{ }^{\mathrm{PM}}\right)$.

Observa-se na Figura 2 que todos os métodos de determinação do Kt, com exceção do proposto por Bernardo et al. (1996), apresentaram coeficientes angulares (b) abaixo de 1, indicando tendência de superestimativa da ETo estimada pelo método considerado como padrão, assim como ocorreu para os valores médios mensais. Mendonça et al. (2003) estudando metodologias para estimativa da ETo na região Norte Fluminense, concluíram que o TCA superestimou o método de Penman Monteith $\left(\mathrm{ETo}^{\mathrm{PM}}\right)$.

Observa-se ainda que ocorreu um ajustamento satisfatório em relação aos coeficientes de determinação $\left(\mathrm{R}^{2} \geq 0,80\right)$
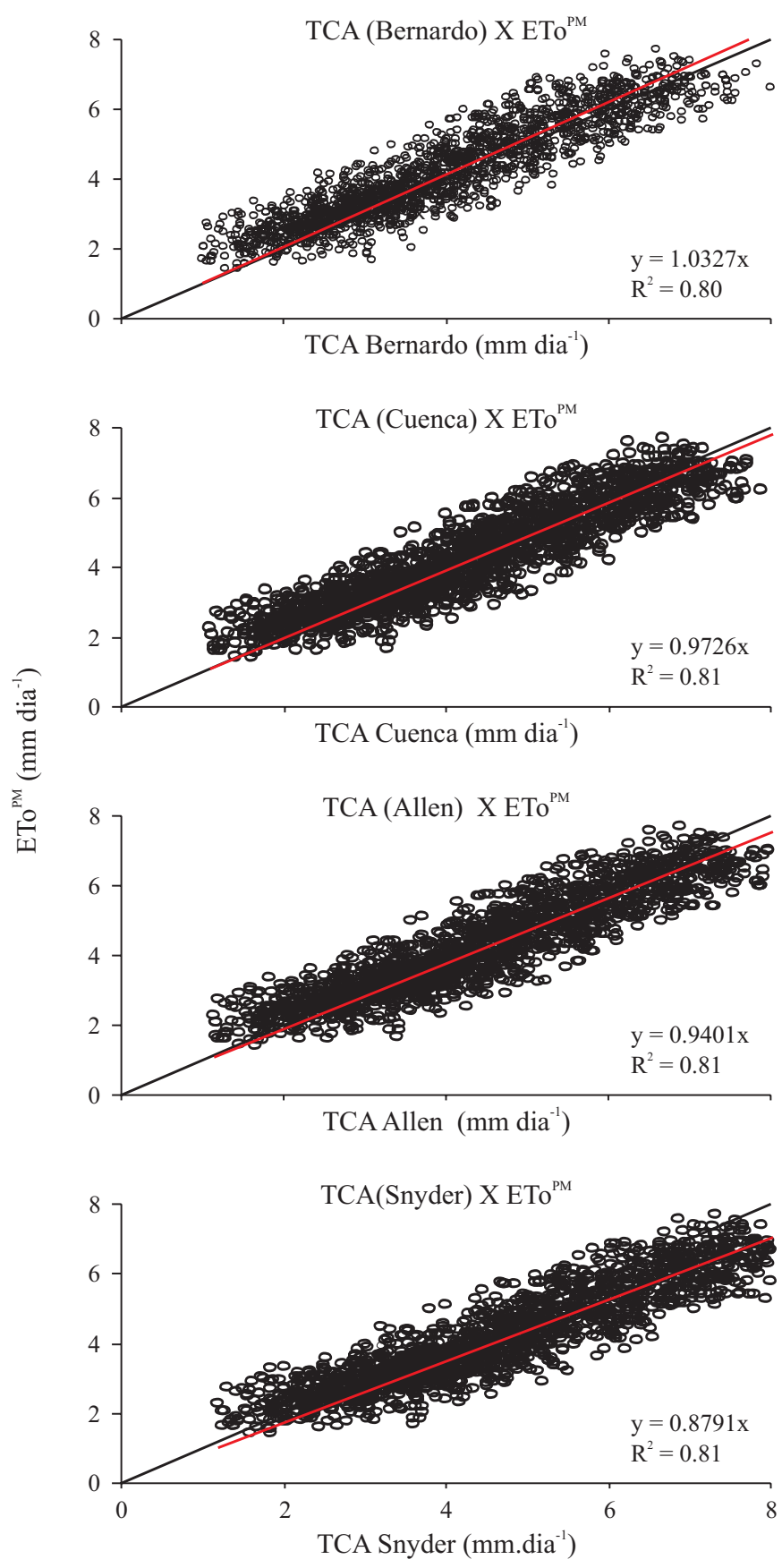

Figura 2. Correlações entre as estimativas diárias da ETo obtidas pelo TCA utilizando as quatro metodologias de obtenção do Kt e a estimada pelo método de Penman Monteith para as metodologias avaliadas, concordando com Carvalho et al. (2006) que comparando métodos para a estimativa da ETo no município de Seropédica, RJ, obtiveram coeficientes com valores semelhantes a estes para o método do TCA. No entanto, a adoção do $\mathrm{R}^{2}$ como único critério de definição da qualidade de métodos não é adequada, uma vez que esse método não estabelece o tipo e a magnitude das diferenças entre um valor padrão e um valor previsto por modelos de estimativa ou outros mecanismos de medida diferentes do padrão (Barros et al. 2009). Deste modo, a analise dos índices de concordância de Wilmont (D), do erro médio absoluto (EMA), do erro máximo (EMAX) e da eficiência dos modelos (EF) auxiliam a interpretação dos resultados.

Na Tabela 2 são apresentados os resultados obtidos com a análise estatística dos dados.

Tabela 2. Valores do coeficiente angular (b), coeficiente de determinação $\left(R^{2}\right)$, índice de concordância de W ilmott (D), erro médio absoluto (EMA em $\mathrm{mm} \mathrm{d}^{-1}$ ), eficiência do método (EF) e erro máximo absoluto (EMAX em $\mathrm{mm} \mathrm{d}^{-1}$ )

\begin{tabular}{lcccccc}
\hline Metodologias & $\mathbf{B}$ & $\mathbf{R}^{2}$ & $\mathbf{D}$ & EF & EMA & EMAX \\
Bernardo et al. (1996) & 1,03 & 0,80 & 0,95 & 0,79 & 0,53 & 1,78 \\
Allen et al. (1998) & 0,94 & 0,81 & 0,95 & 0,77 & 0,53 & 2,14 \\
Cuenca (1989) & 0,97 & 0,81 & 0,95 & 0,81 & 0,50 & 1,83 \\
Snyder (1992) & 0,88 & 0,81 & 0,92 & 0,63 & 0,69 & 2,68 \\
\hline
\end{tabular}

Observa-se na Tabela 2 que a metodologia de maior eficiência para determinação do Kt e posterior conversão da evaporação do TCA em ETo diária, foi o proposto por Cuenca (1989) $(E F=0,81)$, seguido do método proposto por Bernardo et al. (1996) ( $\mathrm{EF}=0,79)$. Estes resultados concordam com Sentelhas \& Folegatti (2003) e Mendonça et al. (2006); contudo, em relação ao índice de concordância de Wilmontt (D), que mede a concordância ou similaridade entre os métodos, houve um ajustamento bastante homogêneo entre as metodologias avaliadas, todas com $\mathrm{D} \geq 0,92$.

O valor fixo proposto por Bernardo et al. (1996) para a região do Norte Fluminense e a metodologia proposta por Allen et al. (1998) apresentaram o mesmo erro médio absoluto (EMA $=0,53 \mathrm{~mm} \mathrm{~d}^{-1}$ ) e eficiências bastante próximas ( $E F=0,79$ e 0,77 respectivamente). O menor erro médio absoluto (EMA $=0,50 \mathrm{~mm} \mathrm{~d}^{-1}$ ) foi obtido pela metodologia de Cuenca (1989), que também apresentou o segundo menor valor do erro máximo $\left(\mathrm{EMAX}=1,83 \mathrm{~mm} \mathrm{~d}^{-1}\right)$.

A metodologia de Snyder (1992) além de ter apresentado o maior erro médio absoluto (EMA $=0,69 \mathrm{~mm} \mathrm{~d}^{-1}$ ) foi a que também apresentou o maior erro máximo (EMAX $=2,68 \mathrm{~mm} \mathrm{~d}^{-1}$ ) e a menor eficiência $(E F=0,63)$. Braga et al. (2008) estudando metodologias para a determinação do Kt, no Vale Submédio São Francisco, Bahia, encontraram que para suas condições climáticas a metodologia de Snyder (1992) superestima a ETo, não sendo a mais adequada para a sua região. Vescove \& Turco (2005) comparando métodos de estimativa de ETo utilizando a metodologia proposta por Snyder (1992) para a determinação do Kt, não constataram ajustamento satisfatório entre os dois métodos, ocorrendo dispersão dos dados, com resultado similar ao encontrado neste trabalho. Estes resultados diferem do 
encontrado por Conceição (2002), que avaliou a metodologia de Snyder (1992) como a melhor para as condições climáticas do Noroeste de São Paulo para obtenção do Kt.

\section{CONCLUSÕES}

1. A melhor metodologia encontrada para o calculo do Kt utilizado pelo método do tanque Classe A, em Campos dos Goytacazes, RJ, foi a proposta por Cuenca (1989), obtendo a maior eficiência.

2. Na ausência de dados meteorológicos, a metodologia proposta por Bernardo et al. (1996) poderá ser utilizada.

3. O método de Snyder (1992) não apresentou desempenho satisfatório para a estimativa da ETo em Campos dos Goytacazes, RJ.

\section{LITERATURA CITADA}

Allen, R. G.; Pereira, L. S.; Raes, D.; Smith, M. Crop Evapotranspiration: guidelines for computing crop requirements. Rome: FAO, 1998. 301p. FAO Irrigation and Drainage Paper 56

Barros, V. R.; Souza, A. P.; Fonseca, D. C.; Silva, L. B. D. Avaliação da evapotranspiração de referência na Região de Seropédica, Rio de Janeiro, utilizando lisímetro de pesagem e modelos matemáticos. Revista Brasileira de Ciências Agrárias, v.4, n.2, p.198-203, 2009.

Bernardo, S.; Sousa, E. F.; Carvalho, J. A. Estimativa da evapotranspiração potencial de referência (ETo) para as "Áreas de Baixada e Tabuleiros” da Região Norte Fluminense, Campos dos Goytacazes: UENF, 1996. 14p. Boletim Técnico n.1

Borges, A. C.; Mendiondo, E. M. Comparação entre equações empíricas para estimativa da evapotranspiração de referência na Bacia do Rio Jacupiranga. Revista Brasileira de Engenharia Agrícola Ambiental, v.11, n.3, p.293-300, 2007.

Braga, M. B.; Calgaro, M., Moura, M. S. B.; Silva, T. G. F. Coeficientes do tanque classe "A" para estimativa da evapotranspiração de referência na região do Vale do Submédio São Francisco, estado da Bahia. Revista Brasileira de Agrometeorologia, v.16, n.1, p.49-57, 2008.
Carvalho, D. F.; Silva, L. D. B.; Folegatti, M. V.; Costa, J. R.; Cruz, F. A. Avaliação da evapotranspiração de referência na região de Seropédica-RJ, utilizando lisimetro de pesagem. Revista Brasileira de Agrometeorologia, v.14, n.2, p.187-195, 2006.

Conceição, M. A. F. Reference evapotranspiration based on class A pan evaporation. Scientia Agricola, v.59, n.3, p.417-420, 2002.

Cuenca, R. H. Irrigation system design: A engineering approach. New Jersey: Pretince-Hall, Englewood Cliffis, 1989. 133p.

Duarte, W. O.; Barros, D. D. L.; Assunção, W. L. Comparação entre as leituras diárias do tanque classe "A" e o evaporímetro de piché, da estação climatológica da Universidade Federal de Uberlândia. In: Simpósio Regional de Geografia: Perspectivas para o Cerrado no Século XXI, 2, 2003, Uberlândia. Anais... Uberlândia: UFU, 2003. p.1-5.

Mendonça, J. C.; Sousa, E. F.; Andre, R. G. B.; Bernardo, S. Coeficientes do tanque classe A para estimativa de evapotranspiração de referência em Campos dos Goytacazes, RJ. Revista Brasileira de Agrometeorologia, v.14, n.1, p.123-128, 2006.

Mendonça, J. C.; Sousa, E. F.; Bernardo, S.; Dias, G. P.; Grippa, S. Comparação entre métodos de estimativa da evapotranspiração de referência (ETo) na região Norte Fluminense, RJ. Revista Brasileira de Engenharia Agrícola e Ambiental, v.7, n.2, p.275-279, 2003.

Oliveira, R. A.; Tagliaferre, C.; Sediyama, G. C.; Materam, F. J. V.; Cecon, P. R. Desempenho do Irrigâmetro na estimativa da evapotranspiração de referência. Revista Brasileira de Engenharia Agrícola Ambiental, v.12, n.2, p.166-173, 2008.

Sentelhas, P. C.; Folegatti, M. V. Class A pan coefficients (Kp) to estimate daily reference evapotranspiration (ETo). Revista Brasileira de Engenharia Agrícola e Ambiental, v.7, n.1, p.111-115, 2003.

Silva, L. C.; Rao, T. V. R. Avaliação de métodos para estimativa de coeficientes da /cultura de amendoim. Revista Brasileira de Engenharia Agrícola e Ambiental, v.10, n.1, p.128-131, 2006.

Silva, V. P. R.; Belo Filho, A. F.; Silva, B. B.; Campos, J. H. B. C. Desenvolvimento de um sistema de estimativa da evapotranspiração de referência. Revista Brasileira de Engenharia Agrícola e Ambiental, v.9, n.4, p.547-553, 2005.

Snyder, R. L. Equation for evaporation pan to evapotranspiration conversion. Journal of Irrigation and Drainage Engineering of ASCE, v.118, n.6, p.977-980, 1992.

Vescove, H. V.; Turco, J. E. P. Comparação de três métodos de estimativa da evapotranspiração de referência para região de Araraquara, SP. Engenharia Agrícola, v.25, n.3, p.713-721, 2005. 